\title{
Wireless Photoplethysmography Sensor for Continuous Blood Pressure Biosignal Shape Acquisition
}

\author{
C. Bambang Dwi Kuncoro, ${ }^{1,2}$ Win-Jet Luo, ${ }^{2}$ and Yean-Der Kuan $\mathbb{D}^{3}$ \\ ${ }^{1}$ Electrical and Instrumentation Laboratory, Politeknik Negeri Bandung, Jl. Gegerkalong Hilir, Ds. Ciwaruga, Bandung, \\ 40012 Jawa Barat, Indonesia \\ ${ }^{2}$ Graduate Institute of Precision Manufacturing, National Chin-Yi University of Technology, No. 57, Sec. 2, Zhongshan Rd., \\ Taiping Dist., Taichung City 41170, Taiwan \\ ${ }^{3}$ Department of Refrigeration, Air Conditioning and Energy Engineering, National Chin-Yi University of Technology, No. 57, Sec. 2, \\ Zhongshan Rd., Taiping Dist., Taichung City 41170, Taiwan
}

Correspondence should be addressed to Yean-Der Kuan; ydkuan@ncut.edu.tw

Received 8 August 2019; Accepted 8 January 2020; Published 24 February 2020

Guest Editor: Jurij Tasic

Copyright (c) 2020 C. Bambang Dwi Kuncoro et al. This is an open access article distributed under the Creative Commons Attribution License, which permits unrestricted use, distribution, and reproduction in any medium, provided the original work is properly cited.

\begin{abstract}
Blood pressure assessment plays a vital role in day-to-day clinical diagnosis procedures as well as personal monitoring. Thus, blood pressure monitoring devices must afford convenience and be easy to use with no side effects on the user. This paper presents a compact, economical, power-efficient, and convenient wireless plethysmography sensor for real-time blood pressure biosignal monitoring. The proposed sensor facilitates blood pressure signal shape sensing, signal conditioning, and data conversion as well as its wireless transmission to a monitoring terminal. Received data can, subsequently, be compiled and stored on a computer via a Wi-Fi module. During monitoring, users can observe blood pressure signals being processed and displayed on the graphical user interface (GUI) - developed using a virtual instrumentation (VI) application. The proposed device comprises a finger clip optical pulse sensor, analogue signal preprocessing, microcontroller, and Wi-Fi module. It consumes approximately $500 \mathrm{~mW}$ power when operating in the active mode and synthesized using commercial off-the-shelf (COTS) components. Experimental results reveal that the proposed device is reliable and facilitates efficient blood pressure monitoring. The proposed wireless photoplethysmographic (PPG) sensor is a preliminary (or first) version of the intended device manifestation. It provides raw blood pressure data for further classification. Additionally, the collected data concerning the blood pressure wave shape can be easily analysed for use in other biosignal observations, interpretations, and investigations. The design approach also allows the device to be built into a wearable system for further research purposes.
\end{abstract}

\section{Introduction}

Several innovations have led to significant advances in healthcare systems by enhancing both functionality and capability of different monitoring systems. Acute, precarious diseases can be diagnosed at their early stages using new-age electronic equipment. Modern diagnostic and therapeutic approaches bear high operation costs and require expensive equipment.

Moreover, in recent years, the advent of wearable technology has afforded accurate recording and precise processing of biosignals. Blood pressure is a vital physiological parameter that indicates the functional well-being of the cardiovascular system [1]. Conventional blood pressuremonitoring devices are either limited in scope with regard to systolic and diastolic blood pressure measurements [2] or unreliable and uncomfortable from the viewpoint of prolonged use. Therefore, there exists the need for a noninvasive, ambulatory care system for long-term blood pressure monitoring while accounting for details concerning blood pressure variability. Availability of such a system would facilitate easy and timely prediction of serious cardiovascular diseases during antihypertensive therapy sessions [3]. 
During development of psychophysiology-monitoring systems, it is important to consider aspects, such as convenience, comfort, and minimum side effects on the user. Such systems detect and record physiological biosignals of patients through a sensor attached to their body. Although the said signals are stored and processed locally, monitoring systems must comprise a wireless module to allow effective signal transmission as and when required. A wearable biosignal monitoring instrument is preferred owing its afforded comfort and convenience of use. Moreover, embedded sensors in such devices can be placed directly in contact with the user's (patient's) body, and their pulse can be recorded. Wearable technology has proven to be effective and promising in medical and clinical applications owing to its noninvasive, comfortable, and accurate measurement techniques.

Several researchers have proposed numerous methods to collect and analyse psychophysiological biosignals. An extant study [4] proposed a mechanism to sense blood volume and pulse signals from fingertips using transmissionmode photoplethysmography. The proposed sensor was sensitive, economic, and consumed less power. It employed a light-emitting diode as a transmitter and a phototransistor as detector. A microcontroller processes signals obtained from the sensor. Advantages of this mechanism were costeffectiveness, compactness, and reduced power consumption.

Ghamari et al. [5] proposed a wireless PPG device enclosed in a wristband-type unit, wherein arterial-pulse data were collected using a sensor, and the same were analysed by the said device. The said device comprised an optical sensor, signal conditioning unit, microcontroller, and Bluetooth module. Collected pulse data were monitored, filtered, amplified, processed, and transferred to another smart device. Additionally, the authors proposed a model using two Gaussian functions to describe photoplethysmographic signals.

Shimazaki and Hara [6] proposed a heart rate- (HR-) sensing device with motion artefact (MA) cancellation along with PPG and MA sensors [6]. The said device was claimed to produce accurate results even in cases of vigorous physical exercise owing to use of two sensors-HR sensor fitted to subjects' waist (back) and a device (Holter monitor) fitted to their chests to monitor ECG waveforms. The authors described the MA cancellation principle in detail, albeit they did not discuss adequate values of relevant design parameters.

Gothwal [7] proposed an economic, wearable PPG system using available components. The device comprised an optical sensor (IR transmitter and receiver), preprocessing unit (buffer amplifier, two-stage band pass filter, amplifier, and comparator), processor (microcontroller), and display unit. Biosignals acquired from subjects' fingers were provided as input to the IR transmitter and receiver. To avoid excessive loading, acquired signals were passed through the buffer amplifier using a coupling mechanism between the IR-receiver output and bandpass-filter input. Subsequently, the output from the buffer amplifier was processed using the microcontroller.

In another study [8], a heart rate monitor (HRM) based on the reflectance photoplethysmography technique was developed to sense pulses from subjects' fingertips. Sensed pulse signals were filtered and amplified using a two-stage operational amplifier (Op-Amp), and the same were processed using a microcontroller. The recorded heart rate was displayed on a liquid-crystal display (LCD) in beats per minute (BPM). The proposed device was claimed to be convenient, inexpensive, and portable compared with the competition. Athletes could use this device to monitor their heart rate with minimal errors.

Zangróniz et al. [9] introduced a wearable photoplethysmography sensor capable of assessing mental distress. The proposed design employed optical plethysmograms to obtain blood volume information using an appropriate sensor. Additionally, a discriminant tree-based model was developed to determine parameter dependencies. Assessment of the proposed model revealed an overall accuracy of $82.35 \%$.

Similarly, a multipurpose photoplethysmographic sensor was proposed in [10] to detect multiwavelength photoplethysmographs by penetrating skin at various depths. The proposed device was named "SmartPPG," and it could operate under transmission and reflection modes with the transmission mode being confined to thin body parts-fingers and earlobes. A light source was used to illuminate parts of the skin under observation, thereby reflecting photoplethysmographs. Operation in the reflection mode optimized both the light wavelength and distance between its source and photodetector.

Botman et al. [11] developed a device to monitor cardiovascular activity. The device is dedicated working in an automated workstation for data collection and analysis in large-scale medical measurements. The proposed design was based on a PPG sensor attached to body parts, such as the nose-bridge, earlobe, fingers, and wrist, to facilitate evaluation of physiological parameters, such as the heart rate and pulse-wave characteristics.

Leier et al. [12] proposed use of a smart optical sensor to monitor different tissue layers constituting the cardiovascular system. Based on acquired PPG signals, the proposed sensor could extract physiological parameters, such as the heart and breath rates, skin microcirculation dynamics, oxygen saturation, and vasomotion activity. The said sensor could be attached to patient bodies at four different points depending on their distance from the four photodetectors. The system comprised 32 light-emitting sources capable of emission at four different wavelengths. All electronic components, including LEDs and photodiodes, were moulded using medicalgrade silicone to facilitate ease and comfort of wearability.

Ishikawa et al. [13] presented a wristband-type PPG heart rate sensor [13] capable of detecting heart rate variability. Additionally, the device was equipped with a motion artefact cancellation framework to handle motion artefacts during daily activities. During assessment of the proposed sensor operation, activities of the arm, finger, and wrist were observed to cause significantly negligible motion artefacts. Additionally, assessment results revealed that the proposed device and coupled motion cancellation framework can be continuously used to monitor heart rate variability. Moreover, the device could be used to recognize and analyse emotions.

Spigulis et al. [14] developed a wireless PPG sensor combining GaAs-radiation emitting and Si-photon diodes placed adjacent to each other to imitate PPG reflection. A 
microcontroller processed sampled signals at programmable sampling frequencies (e.g., $100 \mathrm{~Hz}$ ) using a 10-bit analogueto-digital converter. Processed signals were subsequently transmitted in real time via a Bluetooth module within $10 \mathrm{~m}$ range.

Abovementioned extant researches focus primarily on heart rate analysis using PPG sensors followed by local display of monitoring results on the device-LCD. While some extant studies allow data to be stored on external storage devices, others support data transmission to portable monitoring devices via direct (USB and/or SPI interfaces) or wireless (Bluetooth or GPRS) connections. Any further processing of raw data can be performed post their collection. Additionally, there exist commercial products that can be directly employed in clinical and psychophysiological applications [15-17]; however, these products do not provide users access to raw data.

This paper presents a device capable of recording blood pressure biosignal shapes for psychophysiological and clinical purposes. The proposed device incorporates a $\mathrm{Wi}-\mathrm{Fi}$ module (ISM-Industry, Scientific, Medical band) for transmitting, displaying, and storing acquired biosignal data at a monitoring terminal (computer). Wave shapes used in this study were acquired from healthy volunteers, and acquired data was continuously monitored in real-time on an interactive GUI while simultaneously being used for blood pressure monitoring. Collected data concerning the blood pressure wave shape can be easily analysed for use in other biosignal observations, interpretations, and investigations. The device is aimed at maximizing comfort, convenience, affordability, and compactness while minimizing side effects (if any), operational costs, and power consumption. The proposed wireless PPG sensor is a preliminary (or first) version of the intended device manifestation. It provides raw blood pressure data for further classification, and the design approach allows the device to be built into a wearable system for further research purposes.

\section{Materials and Methods}

2.1. Photoplethysmography (PPG). The term "photoplethysmography" is derived from the Greek word "plethysmos," which means "to increase." As mentioned in [18], plethysmography means "[...] finding variations in the size of a body part owing to variations in the amount of blood passing through or contained in that body part." Pulsatile tissue volumes can be measured using conventional plethysmographs, such as strain gauges, capable of measuring changes under extreme conditions. This technique can be applied to all blood vessels to determine their overall change in volume. Arterial pulsations are the most significant, whereas capillaries are quite noncompliant because they exclusively record minor pulsations. Venous oscillations might occur depending on the measurement technique, albeit such oscillations are often cancelled under application of external pressure [19]. Arterial blood pressure can be measured indirectly using a plethysmogram [20].

Photoplethysmography is similar to traditional plethysmography, albeit not identical. When operating in the

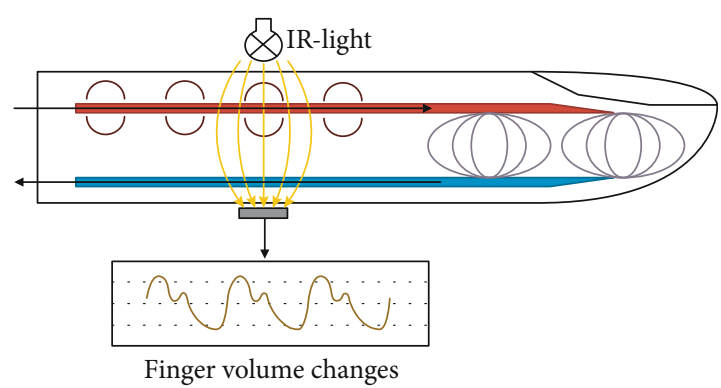

Figure 1: Principle of photoplethysmography [21].

transmission mode, PPG devices use an LED operating on one side of the tissue and a photodetector on the other to demonstrate the obstruction and absorption of incident light. If both the LED and photodetector are placed adjacent to each other, all incident lights may reflect off the tissue surface. Plethysmographic devices cannot measure blood pressure; however, they can evaluate changes in the blood volume. The plethysmographic principle is depicted in Figure 1.

Volumetric changes in blood vessels were first reported by Hertzman [22] using the term "photoplethysmography," and they described two plethysmograph signal components-pulsatile (volume pulse) and baseline (blood volume). Accordingly, plethysmograph signals comprising alternating current $(\mathrm{AC})$ components are indicative of the total absorbance owing to the arterial blood pulsatile component, whereas those comprising direct current (DC) components reveal absorbance caused by other nonpulsatile components as well as the constant flow of arterial and venous blood. Typical plethysmograph signals are depicted in Figure 2.

Absolute changes in blood volume can be accurately measured using the chamber-plethysmography method, wherein volumetric changes $(d V / d t)$ can be transformed into blood flow $(F)$ using the relation $F=d V / d t$. However, some cases only concern relative volumes. Such cases provide information pertaining to time exclusively without any consideration of the amplitude or signal shape. In such cases, electrical impedance plethysmography or photoplethysmography can help provide required information. A literature review regarding plethysmography has been reported in [23], and a more comprehensive review concerning PPG can be found in [24].

Arterial blood pressure is the main cause of PPG signal variations, and as described in Figure 3, the PPG dependence on arterial blood pressure is similar to that of true plethysmograms $[19,25]$.

2.2. Proposed System. A schematic of the wireless photoplethysmography sensor design proposed in this paper is depicted in Figure 4. Acquired PPG signals are first provided as input to a microcontroller through a signal conditioning (filter and amplifier) circuit. Data obtained is subsequently analysed and organised into packets suitable for transmission over a Wi-Fi network through a universal asynchronous receiver transmitter (UART). Data received by the Wi-Fi 


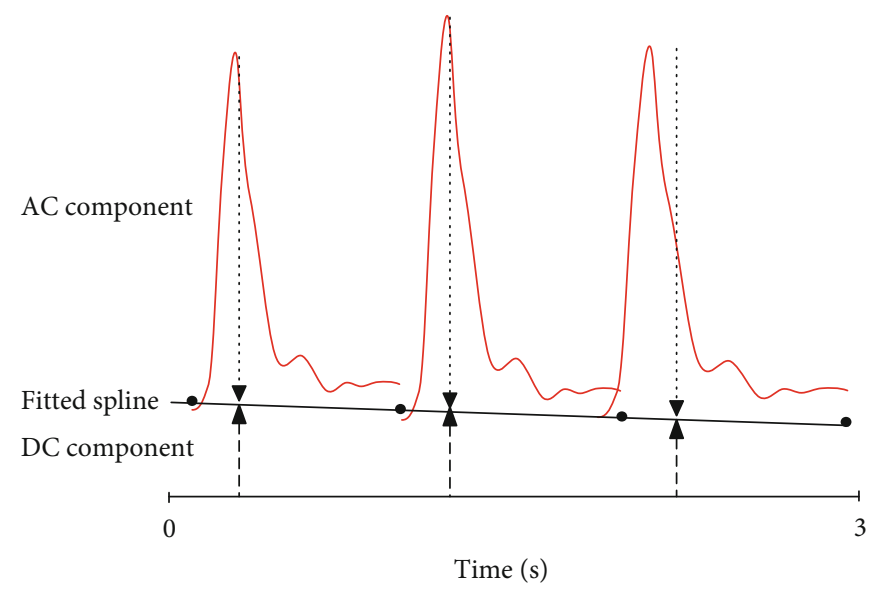

FIGURE 2: AC and DC plethysmograph signals.

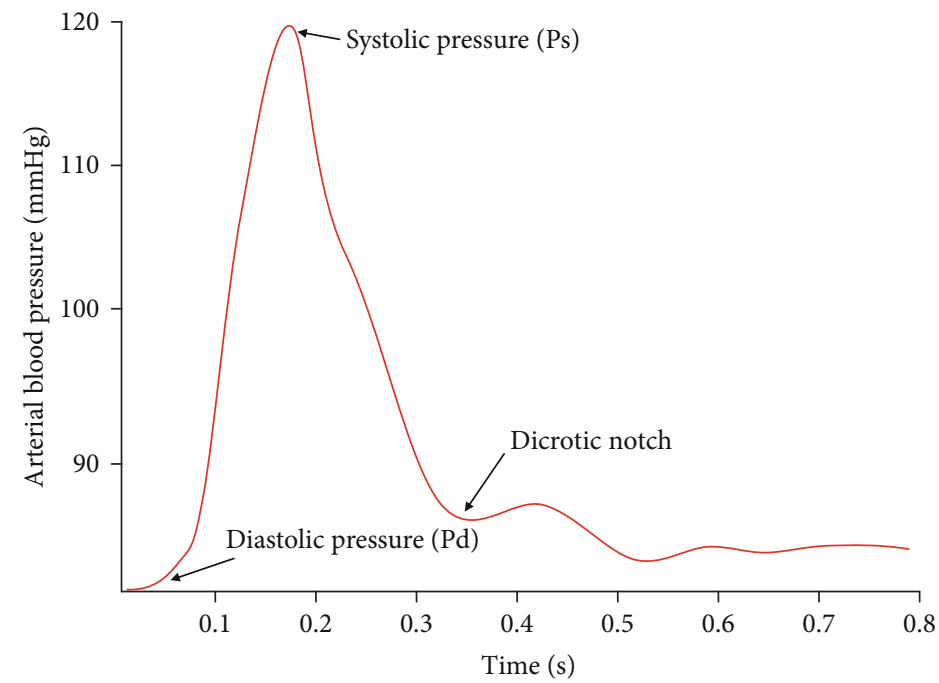

Figure 3: Radial arterial blood pressure waveform cycle (AC component).

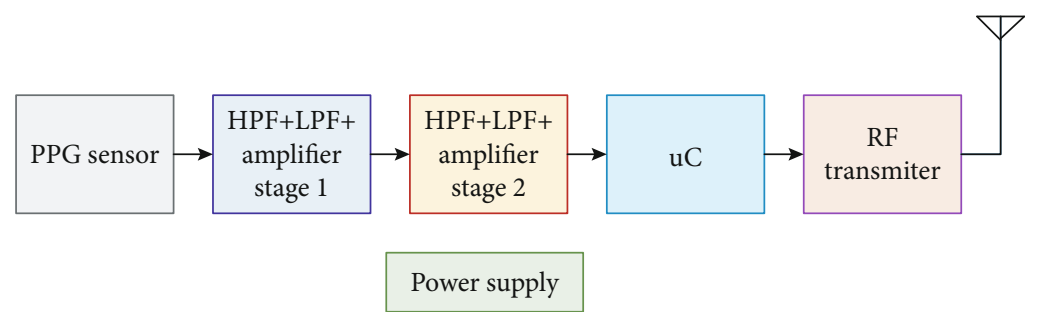

FIgURE 4: Block diagram of wireless PPG sensor.

module is transmitted to a computer where it can be displayed and/or stored.

An LED placed at one end of a finger emits infrared light through the skin. Arterial blood partially absorbs this emitted light which changes according to the pulse. The photodetector placed at the other end of the finger receives any nonabsorbed light and generates an associated continuous pulse signal.

A two-stage analogue preprocessing block filters and amplifies signals received from the sensor. The first stage comprises a passive high-pass filter (HPF) with cut-off frequency $f_{\mathrm{c}}=0.7 \mathrm{~Hz}$ intended to eliminate DC signal components. Additionally, an active low-pass filter (LPF) with constant gain and cut-off frequency of 101 and $2.34 \mathrm{~Hz}$, respectively, was incorporated in the first stage. The said HPF-LPF combination eliminates unwanted DC signal components, thereby reducing noise interference above $60 \mathrm{~Hz}$. The configuration of the second stage is identical to that of the first stage. These filter (first) and amplifier (second) stages modify the PPG sensor signal close to the TTL 


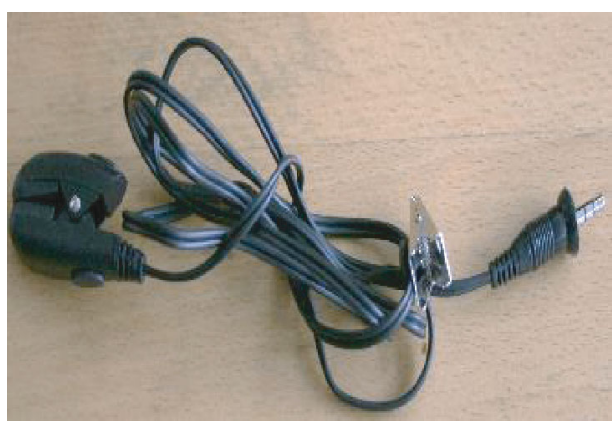

(a)

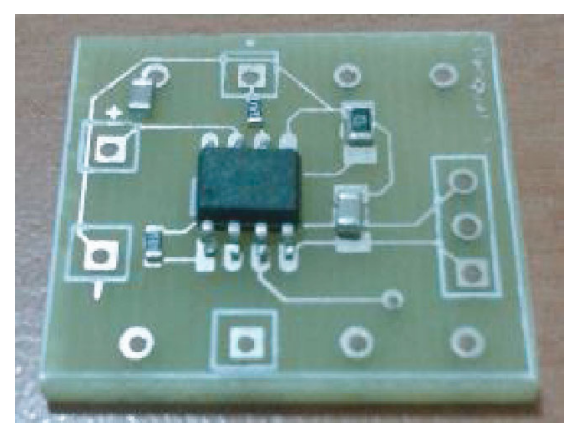

(b)

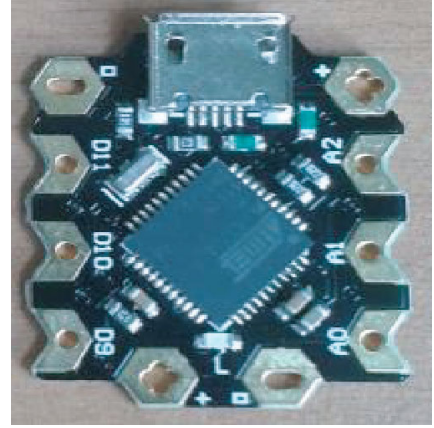

(c)

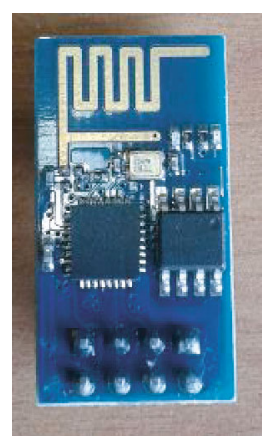

(d)

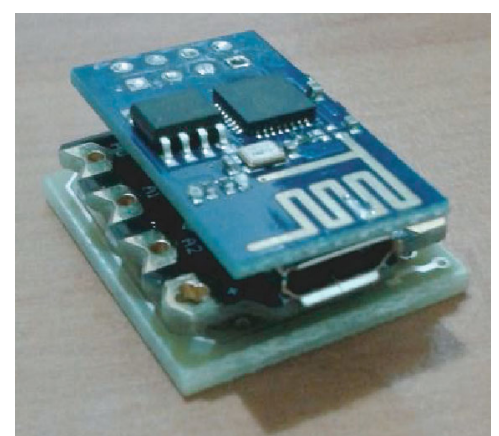

(e)

FIgURE 5: Developed devices. (a) Finger clip optical pulse sensor. (b) Analogue preprocessing. (c) Arduino Beetle board. (d) ESP8266 Wi-Fi module. (e) Wireless PPG sensor module implementation.

(transistor-transistor logic) pulse, which is synchronized with the heart rate.

The microcontroller forms the main component of the wireless PPG sensor, since it serves as an integrated data acquisition system incorporating several features, including low power consumption, compact size, and low cost. It converts analogue skin impedance signals to their digital equivalents prior to processing them. Additionally, the microcontroller communicates with the wireless block through the UART serial port.

The wireless block performs RF communication between the PPG sensor and a remote receiver via $\mathrm{Wi}-\mathrm{Fi}$ connectivity. Collected data is relayed over a UART serial port to a Wi-Fi module, which transmits the same to a laptop or a personal computer (PC). The Wi-Fi module was selected owing to its free bandwidth and wide operating range (typically $50 \mathrm{~m}$ ). The power-supply block dissipates power to other components at the required supply voltage, which is regulated to achieve optimum device functionality.

\section{Results and Discussion}

3.1. Device Implementation. The proposed setup uses the "finger clips" sensor manufactured by Kyto Electronics (finger pulse sensor HRM-2511B), as depicted in Figure 5(a). Additionally, the monitoring device comprises optoelectronic components, an infrared LED, and a phototransistor. The LED cathode and phototransistor emitter were electrically connected with each other as well as other devices using a standard $3.5 \mathrm{~mm}$ connection jack.
The analogue preprocessing circuit was built using a standard 2-layer FR4 PCB board (1.6 mm thickness), as depicted in Figure 5(b). The PCB schematic and board layout were developed using the Altium Design tool. The PCB board measured $20 \times 22 \mathrm{~mm} \times 1.6 \mathrm{~mm}$. The Microchip operational amplifier-MCP6004-and Maxim Integrated voltage reference-MAX6190-were selected as components of the PPG analogue preprocessing circuit. MCP6004 is a low-power amplifier that exhibits other features favourable for analogue preprocessing, as described in [26]. MAX6190 is a precision, micropower, and low-dropout voltage reference. It has a maximum series-mode bandgap referencing of $35 \mu \mathrm{A}$ quiescent supply current, which makes it ideal for use in combination with battery-powered instruments [27].

An Arduino Beetle board was selected for use in signal processing owing to its ease of use, easy availability, and flexibility with regard to creation of powerful applications while using preexisting libraries. The said Beetle board is depicted in Figure 5(c). Because this is an early stage in the design and development of the intended device, minimum hardware that is both power-efficient and cost-effective was considered herein.

The Arduino Beetle is a minimalized version of the powerful Arduino Leonardo board, albeit it offers identical functionalities. Its key feature is its compactness-it measures only $20 \times 22 \mathrm{~mm}$. Additional features of this board are listed in [28].

For wireless data transmission, a $2.4 \mathrm{GHz}$ ESP8266 Wi-Fi module, depicted in Figure 5(d), was employed in this study. The ESP8266 offers a complete, self-contained Wi-Fi networking solution that can either be applied ad hoc or using 


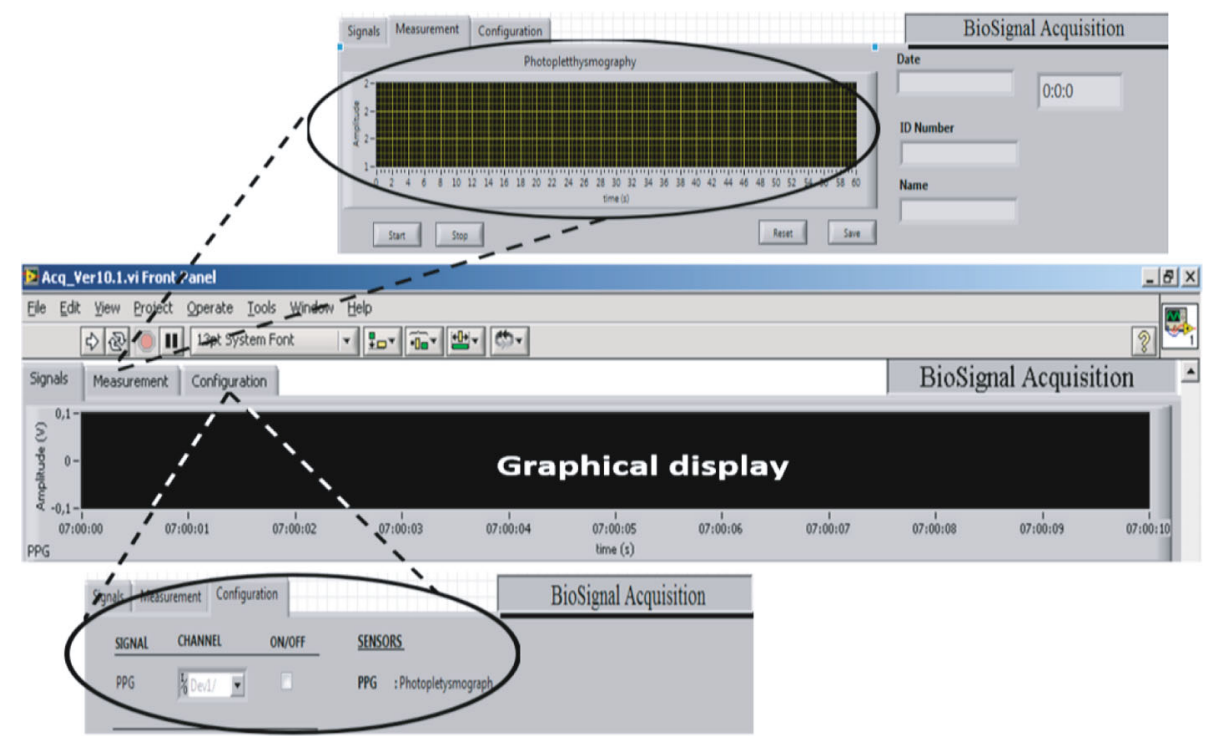

(a)

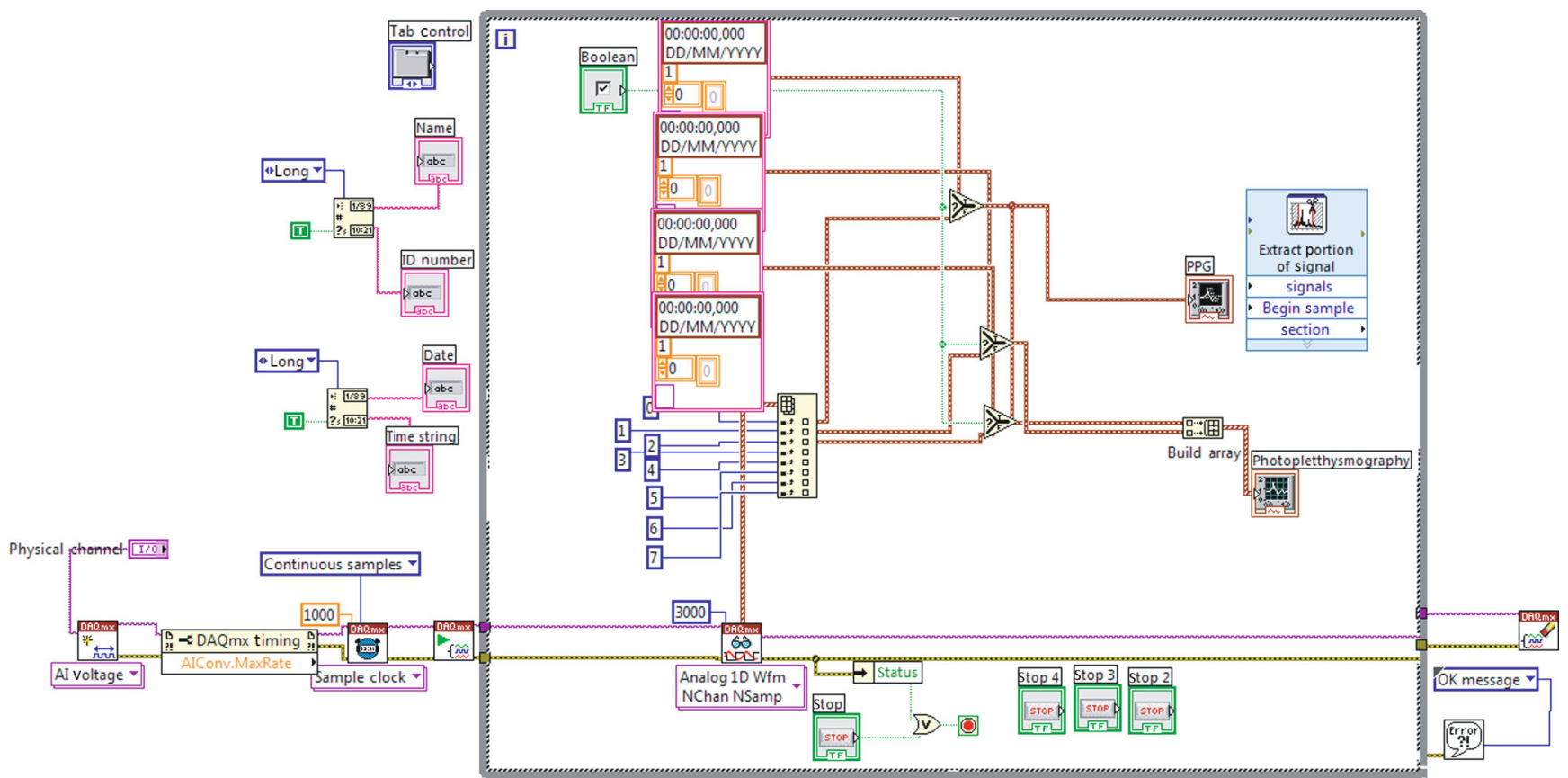

(b)

Figure 6: LabView virtual instrumentation for PPG acquisition. (a) GUI developed in LabView. (b) Signal acquisition algorithm.

a network infrastructure. Additional features concerning the ESP8266 Wi-Fi platform can be found in [29].

Figure 5(e) depicts the first version of the proposed wireless PPG module hardware. As can be seen, the front-end analogue preprocessing unit is interfaced with the Arduino Beetle board and ESP8266 Wi-Fi module. The front-end analogue preprocessing unit was designed to match the size of the Arduino Beetle board. A stackable system was formed with the front-end analogue preprocessing unit, Arduino Beetle, and ESP8266 Wi-Fi module comprising the bottom, middle, and top layers of the system, respectively. All blocks within the device were regulated to operate at $3 \mathrm{~V}$ voltage. The power source employed two $3 \mathrm{~V}$ coin batteries and consumed approximately $500 \mathrm{~mW}$ power when operating in the active mode, which was defined in accordance with the power consumed by main components-the sensor, preamplifier (preamp), microcontroller, and Wi-Fi module [26-30].

3.2. GUI Implementation. The LabView software was used to develop a graphical user interface (GUI) to monitor the shape of blood pressure signals, as illustrated in Figure 6.

Signals obtained from the wireless PPG sensor can be scanned, configured, and examined using the GUI, as depicted in Figure 6(a), which shows variations in the blood pressure signal amplitude (V) against acquisition time (s). In this study, blood pressure signals obtained from different 

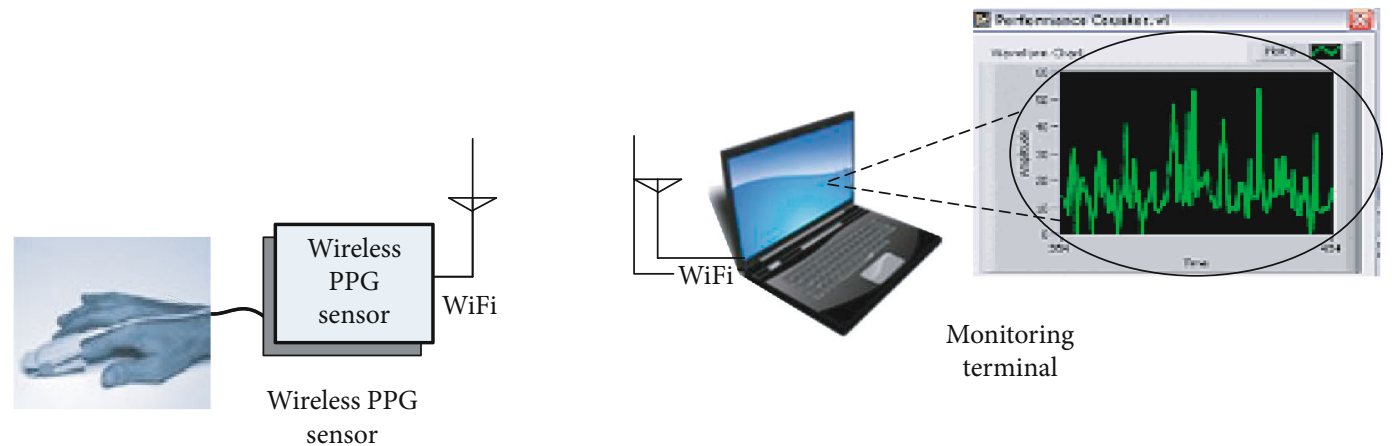

FIGURE 7: Experimental setup used in this study.

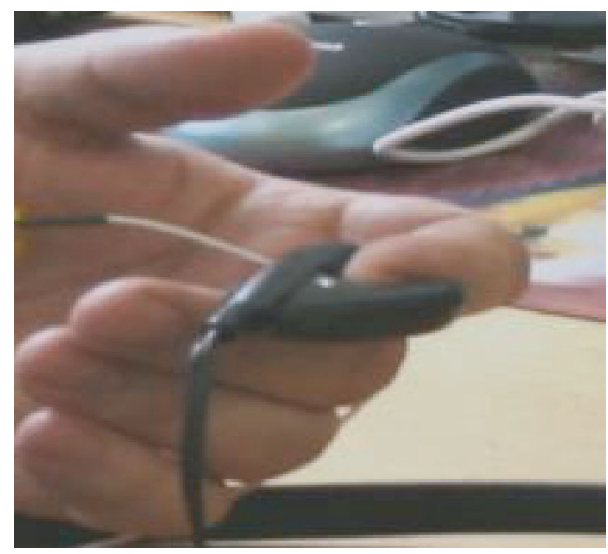

Figure 8: Finger clip pulse sensor.

participants were preprocessed, collected, and stored on a dedicated database. The proposed algorithm continuously acquires and processes raw blood pressure data, as depicted in Figure 6(b), and several toolboxes were employed in conjunction with the LabView software for rapid blood pressure data acquisition and monitoring [31]. Blood pressure signals acquired from participants were stored in a data logger for future psychophysiology and healthcare research purposes.

3.3. Experimental Procedure. During experiments performed in this study, PPG signals were collected using a finger clip instrument; the operation of which is based on the transmission photoplethysmography phenomenon. An IR-LED installed within the finger clip (with approximately $18 \mathrm{~mA}$ current rating) emits infrared waves through the finger, subsequent to which the photodiode performs signal detection, thereafter converting them to corresponding voltage values using a voltage-divider circuit. This generated signal corresponds to the volume of blood flowing within the finger, and therefore, reflects pulse waves generated within blood vessels. Experiments and tests were performed to assess the proposed PPG sensor system. The experimental setup is depicted in Figure 7.

During experiments, the PPG sensor-via the finger clip instrument-was attached to a healthy participant finger to obtain biosignals (Figure 8). Acquired biosignals were transmitted to the processing module for preprocessing, storage, analysis, and organizing data into packets for transmission over the Wi-Fi module. These data packets received by the monitoring terminal (notebook/PC) along with raw PPG biosignals were stored in the data logger, and the same can be viewed in GUI.

3.4. Experimental Results and Discussions. Figure 9 depicts the graphical representation of blood pressure signals. Processed signals can be stored locally on a notebook or personal computer.

Using the above interface (Figure 9), users can observe the shape of their blood pressure signal and associated variation trends in real time during the monitoring experiment. This facilitates early detection of anomalies in blood pressure detection.

Results obtained via assessment of raw blood pressure signals acquired from healthy participants are depicted in Figure 10. Data stored in the data logger during measurement as well as blood pressure signals include artefacts owing to interference of ambient light.

Hertzman [22] defined two important PPG-AC pulse waveform phases-anacrotic (rising edge of a pulse) and catacrotic (falling edge of a pulse). The shape of a blood pressure signal representing the anacrotic and catacrotic phases is depicted in Figure 11 (using the signal from Figure 10 above).

The anacrotic phase denotes the systolic pressure, whereas the catacrotic phase concerns the diastolic pressure and peripheral wave reflections. As depicted in the figure, a dicrotic notch can be observed in the catacrotic phase for participants demonstrating a compliant healthy arterial system.

The PPG signal depicted in Figure 9 can be directly observed during blood pressure measurements with regard to the systolic and diastolic phases. The context of this research is limited to providing raw blood pressure data. However, its scope can be extended to include an analytic method that facilitates estimation of cuffless blood pressure using the PPG signal generated in this research. The regression tree is a suitable analytic approach that can be used for blood pressure estimation [32]. Raw PPG signals require initial processing (preprocessing) to smoothen (i.e., remove signal artefacts) PPG signals. A filter can be employed to perform the said preprocessing, which can subsequently be followed by two-dimensional normalization (width and 


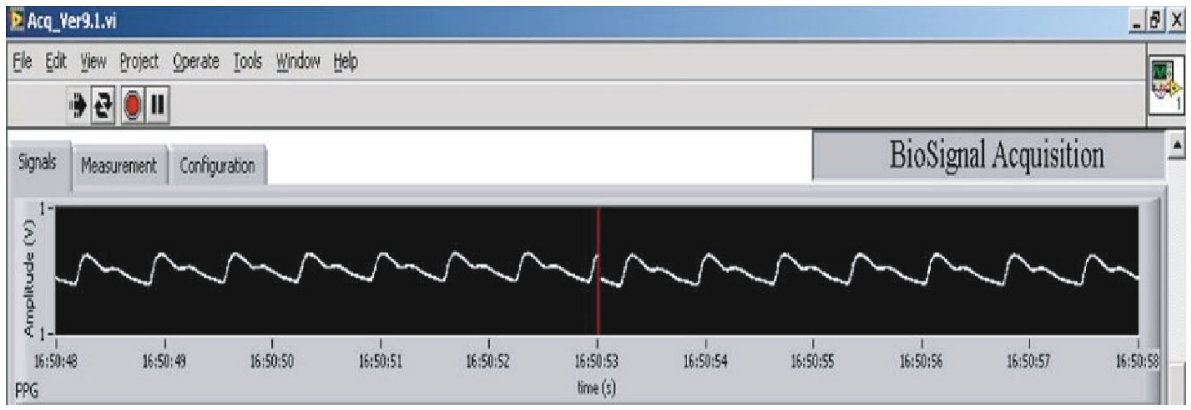

Figure 9: Graphical representation of blood pressure signals.

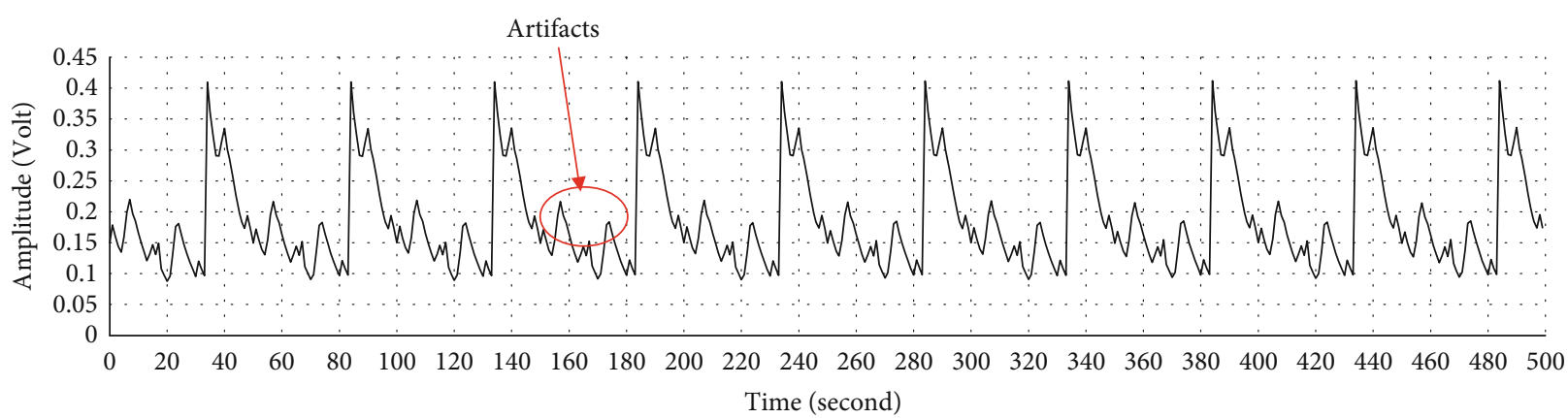

Figure 10: Raw blood pressure signal data obtained via photoplethysmography measurement.

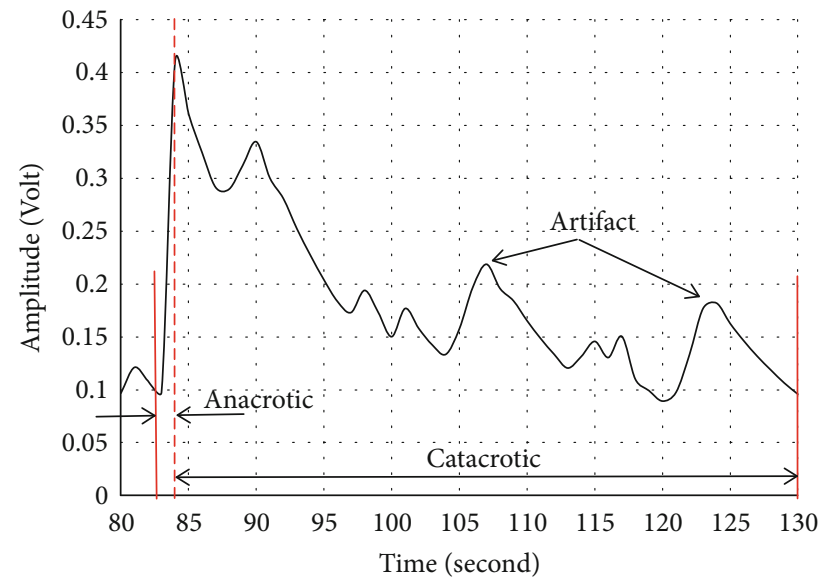

FIgURE 11: Shape of raw blood pressure signal depicting anacrotic and catacrotic phases.

amplitude) of filtered PPG signals. Preprocessed PPG segments comprise pulse area, rising time, and widths of range $25 \%, 50 \%$, and $75 \%$. Thus, the three most significant PPG waveform features are the pulse area, pulse rising time, and pulse width of $25 \%$, which can be further processed using the regression tree.

\section{Conclusions}

This paper presents a simple, compact, inexpensive, powerefficient, comfortable, noninvasive, real-time, and durable wireless photoplethysmograph sensor for monitoring the shape of blood pressure signals. This study implements the first version of the said wireless photoplethysmograph sensor, and initial assessment results (for stationary test scenarios) reveal realisation of reliable blood pressure signal shape monitoring. Additionally, the proposed wireless sensor provides raw blood pressure data that can be utilized for future blood pressure estimations and classifications. In the proposed system, artefacts within PPG signals can be reduced by attaching a Velcro strip around the finger, minimizing ambient light interference, and ensuring accurate placement of the sensor on the participant's finger.

The proposed device in conjunction with a wearable system can be implemented to support further research pertaining to psychophysiology and healthcare applications. As a future endeavour, the authors intend to develop a more convenient and compact wireless PPG wearable sensor capable of acquiring data during physical activities. The said sensor can be combined with an analytic method to accurately estimate and classify blood pressure levels.

\section{Data Availability}

Research data is not available owing to concerns related to participant privacy.

\section{Conflicts of Interest}

The authors declare no conflict of interest. 


\section{Acknowledgments}

The authors would like to thank the Ministry of Science and Technology of Taiwan (MOST 107-3113-E-008-003) for funding this research.

\section{References}

[1] Q. Wang, Y. Wang, Z. Mei, and Z. Liu, "An improved algorithm for non-invasive blood pressure measurement," in International Symposium on Bioelectronics and Bioinformations 2011, pp. 41-44, Suzhou, China, November 2011.

[2] Z. Marcinkevics, M. Greve, J. I. Aivars, R. Erts, and A. H. Zehtabi, "Relationship between arterial pressure and pulse wave velocity using photoplethysmography during the postexercise recovery period," Acta Universitatis Latviensis, vol. 753, pp. 59-68, 2009.

[3] B. P. McGrath, "Ambulatory blood pressure monitoring," The Medical Journal of Australia, vol. 176, no. 12, pp. 588-592, 2002.

[4] V. K. Jayasree, P. J. Shaija, V. P. N. Nampoori, C. P. Girijavallabhan, and P. Radhakrishnan, "A simple and novel integrated opto-electronic system for blood volume pulse sensing and heart rate monitoring," International Journal of Optomechatronics, vol. 1, no. 4, pp. 392-403, 2007.

[5] M. Ghamari, C. Soltanpur, S. Cabrera, R. Romero, R. Martinek, and H. Nazeran, "Design and prototyping of a wristband-type wireless photoplethysmographic device for heart rate variability signal analysis," in 2016 38th Annual International Conference of the IEEE Engineering in Medicine and Biology Society (EMBC), pp. 4967-4970, Orlando, FL, USA, August 2016.

[6] T. Shimazaki and S. Hara, "Design of PPG-based heart rate sensor enabling motion artefact cancellation," Heart, vol. 100, no. 60, p. $20,2015$.

[7] P. Gothwal, "Designing of PPG based heart-beat monitor," International Journal for Research in Applied Science \& Engineering Technology, vol. 4, no. VIII, pp. 86-89, 2016.

[8] K. O. Enalume and A. M. Epemu, "Development of a reflectance photoplethysmogram based heart rate monitoring device," International Journal of Medical Imaging, vol. 5, no. 5, pp. 53-57, 2017.

[9] R. Zangróniz, A. Martínez-Rodrigo, M. López, J. Pastor, and A. Fernández-Caballero, "Estimation of mental distress from photoplethysmography," Applied Sciences, vol. 8, no. 1, p. 69, 2018.

[10] D. U. Uguz, B. Venema, S. Leonhardt, and D. Teichmann, "Multifunctional photoplethysmography sensor design for respiratory and cardiovascular diagnosis," World Congress on Medical Physics and Biomedical Engineering 2018. IFMBE Proceedings, vol 68/2, L. Lhotska, L. Sukupova, I. Lacković, and G. Ibbott, Eds., , pp. 905-909, Springer, Singapore, 2018.

[11] S. Botman, D. Borchevkin, V. Petrov, E. Bogdanov, M. Patrushev, and N. Shusharina, "Photoplethysmographybased device designing for cardiovascular system diagnostics," World Academy of Science, Engineering and Technology International Journal of Medical, Health, Biomedical, Bioengineering and Pharmaceutical Engineering, vol. 9, no. 9, pp. 689-693, 2015.

[12] M. Leier, K. Pilt, D. Karai, and G. Jervan, "Smart photoplethysmographic sensor for pulse wave registration at different vascular depths," in 2015 37th Annual International Conference of the IEEE Engineering in Medicine and Biology Society (EMBC), pp. 1849-1852, Milan, Italy, August 2015.

[13] T. Ishikawa, Y. Hyodo, K. Miyashita, K. Yoshifuji, Y. Komoriya, and Y. Imai, "Wearable motion tolerant PPG sensor for instant heart rate in daily activity," in Proceedings of the 10th International Joint Conference on Biomedical Engineering Systems and Technologies (BIOSTEC 2017), pp. 126-133, Porto, Portugal, 2017.

[14] J. Spigulis, R. Erts, V. Nikiforovs, and E. Kviesis-Kipge, "Wearable wireless photoplethysmography sensors," in Proceedings Volume 6991, Biophotonics: Photonic Solutions for Better Health Care; 69912O, pp. 126-133, Strasbourg, France, May 2008.

[15] Apple Watch series 4June 2019, https://www.apple.com/watch/.

[16] Fitbit Ionic ${ }^{T M}$ WatchJune 2019, https://www.fitbit.com/ionic.

[17] GarminJune 2019, https://www.garmin.com/en-US.

[18] A. Peters, The British Medical Association Illustrated Medical Dictionary, Dorling Kindersley Ltd, London, United Kingdom, 2nd edition, 2007.

[19] A. Reisner, P. A. Shaltis, D. McCombie, and H. H. Asada, "Utility of the photoplethysmogram in circulatory monitoring," Anesthesiology, vol. 108, no. 5, pp. 950-958, 2008.

[20] M. Hirai, S. L. Nielsen, and N. A. Lassen, "Blood pressure measurement of all five fingers by strain gauge plethysmography," Scandinavian Journal of Clinical \& Laboratory Investigation, vol. 36, no. 7, pp. 627-632, 1976.

[21] FiguresJanuary 2019, http://www.cnsystems.at/en/vascularunloading-technique.

[22] A. B. Hertzman, "Observation on the finger volume pulse recorded photoelectrically," American Journal of Physiology, vol. 119, pp. 334-335, 1937.

[23] J. W. Clark, M. R. Neuman, W. H. Olson et al., Medical instrumentation: application and design, Wiley, 1998.

[24] J. Allen, "Photoplethysmography and its application in clinical physiological measurement," Physiological Measurement, vol. 28, no. 3, pp. R1-R39, 2007.

[25] J. Ando, A. Kawarada, M. Shibata, K. Yamakoshi, and A. Kamiya, "Pressure-volume relationships of finger arteries in healthy subjects and patients with coronary atherosclerosis measured non-invasively by photoelectric plethysmography," Japanese Circulation Journal, vol. 55, no. 6, pp. 567-575, 1991.

[26] Microchip operational amplifier MCP6004December 2018, http://ww1.microchip.com/downloads/en/DeviceDoc/21733j .pdf.

[27] Maxim Integrated voltage reference MAX6190December 2019, http://datasheets.maximintegrated.com/en/ds/MAX6190MAX6198.pdf.

[28] Arduino BeetleMarch 2019, http://www.dfrobot.com/wiki/ index.php/Beetle_SKU:DFR0282.

[29] Wi-Fi Module - ESP8266January 2019, https://www.sparkfun .com/products/13252.

[30] PPG_kyto_hrm2511March 2019, http://www.geertlangereis .nl/sensorwiki/doku.php?id=methods:ppg:ppg_kyto_ hrm2511.

[31] J. Olansen and E. Rosow, Virtual Bio-Instrumentation, Biomedical, Clinical and Healthcare Applications in LabVIEW, Prentice Hall PTR, 2002.

[32] S. G. Khalid, J. Zhang, F. Chen, and D. Zheng, "Blood pressure estimation using photoplethysmography only: comparison between different machine learning approaches," Journal of Healthcare Engineering, vol. 2018, 13 pages, 2018. 